\title{
La contabilidad ambiental en empresas industriales de línea blanca en la provincia del Azuay-Ecuador
}

\section{Environmental accounting in white goods industrial companies in the province of Azuay-Ecuador}

\author{
Alexandra Estefanía Sarmiento-Figueroa \\ alexandra.sarmiento49@est.ucacue.edu.ec \\ Universidad Católica de Cuenca, Cuenca- \\ Ecuador \\ https://orcid.org/0000-0003-3223-5624 \\ Diego Vinicio Orellana-Bueno \\ dorellana@ucacue.edu.ec \\ Universidad Católica de Cuenca, Cuenca- \\ Ecuador \\ https://orcid.org/0000-0002-7320-8684 \\ Pablo Javier Perez-Jara \\ pperezj@ucacue.edu.ec \\ Universidad Católica de Cuenca, Cuenca- \\ Ecuador \\ https://orcid.org/0000-0001-8538-5955
}

Recibido: 05 de agosto de 2020 Aprobado: 01 de octubre de 2020 


\title{
RESUMEN
}

La contabilidad ambiental o llamada también contabilidad verde, se define como la presentación de información financiera considerando aspectos ambientales ante un visible deterioro en todo el ecosistema y la falta de actividades humanas amigables con el entorno, su finalidad es la prevención, corrección y la compensación de las secuelas creadas, debido a la problemática generada por las actividades empresariales que tienen efecto dañino en el medio ambiente, para lo cual se realizó una investigación de tipo no experimental, obteniéndose como resultado que la mayoría de las empresas en estudio no aplican la contabilidad ambiental y que requieren de capacitación para la aplicabilidad de la misma, por consiguiente, la presente investigación propone una guía práctica que abarca identificación de costos y gastos ambientales, campañas de uso alternativo de medios de transporte, información medioambiental en los estados financieros e incentivos a los colaboradores que forman parte de actividades ecológicas.

Descriptores: Medio ambiente; impacto ambiental; contabilidad; costes; gasto. (palabras tomadas del Tesauro de la UNESCO).

\begin{abstract}
Environmental accounting or also called green accounting, is defined as the presentation of financial information considering environmental aspects before a visible deterioration in the entire ecosystem and the lack of environmentally friendly human activities, its purpose is the prevention, correction and compensation of the consequences created, due to the problems generated by business activities that have a damaging effect on the environment, for which a non-experimental investigation was carried out, obtaining as a result that most of the companies under study do not apply environmental accounting and that require training for its applicability, therefore, this research proposes a practical guide that covers identification of environmental costs and expenses, campaigns for alternative use of means of transport, environmental information in financial statements and incentives to collaborators who are part of activida ecological des.
\end{abstract}

Descriptors: Environment; environmental impact; accounting; costs; spending. (words taken from the UNESCO Thesaurus). 


\section{INTRODUCCIÓN}

El deterioro ambiental se define como el desgaste del planeta debido al agotamiento de recursos naturales, la destrucción de los ecosistemas y la contaminación; el impacto negativo en el ambiente es evidente y perceptible a los sentidos, los cambios en los diferentes ecosistemas ponen en evidencia la urgente necesidad de articular los planes, programas y políticas entre los Estados, las empresas y los ciudadanos; al respecto, el autor (Díaz, 2019) menciona que la contaminación ambiental es una amenaza a la calidad de vida y a la salud tanto de los seres humanos como de los animales, lo que ha generado conciencia en la acción social, exigiendo a los gobiernos a actuar ante los sectores comerciales, industriales y productivos los que deben comprometerse a preservar el ecosistema con ramas como la economía, la contabilidad, sociología, arquitectura, derecho y muchas otras más disciplinas que han de sumarse a la protección del medio ambiente.

Según lo mencionado en el párrafo anterior, se trae a contexto la contabilidad ambiental también conocida como contabilidad verde o contabilidad ecológica, la misma que, se define como el acto de incluir en la presentación de los estados financieros de cada empresa, elementos que aludan al impacto ambiental que producen sus acciones, con base en este problema se ha visto la necesidad de implementar nuevas medidas para prevenir el deterioro del medio ambiente. Al hablar del sector empresarial, por su naturaleza requiere definir diversas estrategias de medida y moderación ambiental, sin embargo, la filosofía organizacional presenta ciertos límites en cuanto a la contribución de un compromiso ambiental, es decir, las entidades empresariales no asumen una correcta responsabilidad ante la problemática ambiental con el fin de obtener ventajas económicas (Jara, Díaz, \& Morales, 2017).

Por lo expuesto, las organizaciones deben considerar un programa de contabilidad verde en sus informes financieros, esto incluye los bienes, gastos y costos de las inversiones ambientales y otros dispendios significativos relacionados con el desempeño laboral 
(Arguello, 2018). Las empresas requieren de capacitación urgente para contrarrestar la problemática contable-ambiental, en este sentido, se plantea el siguiente problema de investigación ¿Cómo registrar, evaluar y comunicar la actuación medioambiental de las empresas de línea blanca de la provincia del Azuay?, en correspondencia a la problemática, se establece como objetivo diseñar la propuesta de una guía de buenas prácticas contables-ambientales que propicie el adecuado registro, evaluación y comunicación de la actuación medioambiental de las empresas de línea blanca en la provincia del Azuay.

\section{Referencial teórico}

\section{Relación directa entre la contabilidad y su impacto con el medio ambiente}

El término ecología parte de la rama de la biología, que es una ciencia que estudia cómo se relacionan los seres vivos y su coexistencia en el medio ambiente. Es así que, para su estudio cobra un papel fundamental el análisis de la interacción de los seres vivos en el medio en el que habitan, es decir, que todas las actividades que los humanos realizan repercute en el medio ambiente ya sea de manera positiva o negativa; en este sentido, se consideran ciertos factores de estudio como los climáticos, abióticos y bióticos (Caraballo \& Barros, 2011). Por consiguiente, existe una gran importancia de la ecología y educación ambiental, ya que si bien es cierto, educación ambiental no es un término nuevo, al contrario, este término surgió en el año de 1866, sin embargo, en los últimos tiempos está cobrando importancia debido al visible deterioro en todo el ecosistema y la falta de actividades amigables con el medio ambiente por parte de los seres humanos en su vida cotidiana. De igual manera, cobra importancia debido a la necesidad de ayudar a las nuevas generaciones que son las que sufrirán la contaminación ambiental (Sánchez \& Pedrajas, 2010).

Por otro lado, es trascendental destacar que la educación ambiental y la ideología de un mundo sostenible están relacionadas. Esto expresa que no habrá un futuro sostenible sin una apropiada educación ambiental. A raíz de la inadecuada importancia o compromiso 
asignada a la naturaleza, son tan visibles los signos de contaminación global, tal como el agotamiento de recursos naturales, la extinción de algunas especies o pérdida de biodiversidad, la falta de flora-fauna y la pobreza a nivel mundial (Jiliberto, y otros, 2013). En este contexto, la contabilidad es una disciplina que se encarga del estudio y control de los gastos e ingresos que se realizan en las empresas, entidades $u$ organizaciones que facilita la toma de decisiones; de igual manera, la contabilidad parte de la rama de la economía que estudian las partidas de estos sistemas financieros. Entre los principios generales de la contabilidad se puede mencionar que una persona natural y un negocio no deben mezclarse, es así que, los propietarios son acreedores de las empresas que han formado y a pesar de que tengan varias empresas, cada una se trata como una entidad separada, por lo que el propietario es un acreedor más de la entidad, al que en la contabilidad se le representa como capital (Macías \& Roig, 2018).

Las primeras declaraciones de la relación entre la economía y el medio ambiente tuvieron lugar durante los años 1950 a 1960 según lo menciona el autor (Hernández, 2016) derivado de una preocupación referente a las inestabilidades presentadas entre el entorno ambiental y el adelanto económico, del cual emanan los recursos y el soporte de la vida, fue así el sentir de un grupo de economistas de la escuela neoclásica, mientras que, la contabilidad de costos ambientales inicia como un adelanto del tratamiento habitual proporcionado a los costes de producción, añadiéndose varias herramientas como el análisis del ciclo de vida del producto con el objetivo de que se reflejen en el costo real del producto los costos derivados de un trabajo ambiental; este desarrollo en el registro de los costos brinda la información clara y respectiva para una adecuada toma de decisiones, por lo tanto, en el costo final de un producto no se encubren costos o gastos generales.

Existe un término conocido como divorcio contabilidad-medio ambiente, que se refiere a la separación histórica de estos dos; además, se conoce que esto ocurre porque la contabilidad tiene una concepción que se encuentra vinculada al ámbito financiero. En tal 
caso, es necesario extender los conceptos de esta disciplina para estrechar los límites existentes entre estos dos y con la única finalidad de lograr un diálogo interdisciplinario entorno al problema ambiental (Rodríguez, 2011).

Gracias a todos los esfuerzos realizados por varias personas, hoy en día, se han logrado avances en la contabilidad ambiental. Por ejemplo, la contabilidad ambiental se encarga de evaluar daños, explicar las posibles reacciones cuantitativas y cualitativas, tomando un volumen alto de reactivos y activos en controversias (López, Rodríguez, \& Gómez, 2019), en conjunto incluye rubros en los diferentes grupos de cuentas del activo, pasivo y patrimonio así como también del estado de resultados, lo cual permite medir y ser conscientes del impacto de las actividades empresariales en el medio ambiente.

En el periodo de la modernidad se visualiza el mayor esplendor de la ciencia, lo cual implicó una enorme instauración del paradigma científico-mecánico, el mismo que provocó la aparición de otras disciplinas, por lo tanto, surgieron varias de ellas con sus respectivas teorías y se fueron especializando poco a poco cada una, se logró un mayor desarrollo sin duda, sin embargo, condujo a grandes problemáticas ambientales como es la contaminación lo cual repercute de forma directa en la sociedad en general (Rizo \& Collado, 2017).

En este orden de ideas, el manejo de la información contable respecto al medio ambiente es de vital importancia para una eficiente gestión medioambiental en la empresa, dicha información debe ser perceptible en el activo, pasivo, costos y gastos y deben implementarse las cuentas que velen por el cumplimiento medio ambiental, a partir de ello surge la necesidad de disponer de un proceso contable que integre conceptos medioambientales y suministre información acerca de costos ambientales, los cuales deben formar parte de los costos financieros finales (Porras, 2018).

La Organización de las Naciones Unidas (ONU) en 1983 ante la notable desestabilidad y desgaste del medio ambiente, impulsado ante las actividades humanas poco amigables y la producción de las empresas que día a día iban en acelerado crecimiento y ante el 
inminente peligro de supervivencia de la especie humana, implementaron campañas y programas guiados a la protección del medio ambiente en complemento con el crecimiento económico.

Por lo antes expuesto, una de las medidas que controle el efecto dañino que producen las empresas en el medio ambiente es la implementación de información ambiental en la información financiera de cada organización, es así, que se habla de los costos ambientales los mismos que son adicionales a los costos identificados de forma tradicional según la actividad, como lo menciona el autor (Bischhoffshause, 2016) en su artículo y también acentúa importancia en que el nuevo éxito de las empresas está en la gestión adecuada de los costos generados, también de constituir un componente esencial de cualquier sistema de gestión ambiental.

Se hace un llamado a la consciencia empresarial a cerca de las actividades que dichas organizaciones realizan y al impacto que tienen en el medio ambiente de forma directa, además se debe reflejar en los estados financieros el valor de daño ocasionado en el medio ambiente debido a las actividades ejecutadas, así también a manera de ejemplo se puede reflejar en inversión si se desea tomar la vía de estudios medioambientales, con el fin de que se determine que es dañino y que actividades y procesos deben ser transformados y empezar a ser amigables con el medio ambiente.

Así también, se menciona algunos controles que brinda la contabilidad, adicional al control económico realizado con el fin de satisfacer las necesidades de los clientes internos y externos y que presenta la información referente a la gestión, obtención, medición y registro de los recursos naturales, se muestra además otros controles adicionales que son: un control natural, pues es el principal suministro para lograr una producción adecuada, cuidando del uso y distribución de los recursos naturales; un control cultural por cuanto se implementa consciencia en la exposición de la información financiera y un control político debido a la interrelación y a veces contradicciones de los diferentes sectores a los cuales las organizaciones rinden cuentas. 


\section{Adopción de la contabilidad ambiental en empresas de producción}

Los temas ambientales en las últimas décadas han tenido gran impacto en varias entidades, organizaciones mundiales y la sociedad en general, que han evidenciado que los principales responsables del daño ambiental son las industrias y grandes empresas, lo que ha exigido un desarrollo e integración de diversas disciplinas como la contabilidad mediante el tratamiento y aplicación de las Normas Internacionales de Información Financiera (NIIF) emitidas por el International Accounting Standars Board (IASB), varios autores declaran que existe ausencia de un marco normativo y otros recalcan en el desarrollo de una mayor investigación que permita a la contabilidad una adecuada sostenibilidad con un cambio de conciencia por las cuestiones ambientales, en este sentido, se demanda la emisión de nuevas normas que rijan los procedimientos financieros en materia relacionada con el medio ambiente (Luna, 2016).

A este respecto, se resalta la NIIF 6 , que hace referencia a la información financiera relativa a la explotación y la evaluación de recursos minerales, en la misma plantea: mejoras limitadas en las prácticas contables existentes para los desembolsos por exploración y evaluación, así como la comprobación del deterioro de los activos en correspondencia con la NIC 36, y la revelación de información que identifique y explique los importes que en los estados financieros de la entidad surjan de la exploración y evaluación de recursos minerales. Por su parte, la comunidad nacional e internacional tomando en consideración la gran importancia de la responsabilidad medioambiental para lograr el desarrollo de un sistema económico de mercado, determinó que es indispensable contar con políticas ambientales, las mismas que deben ir acompañadas de una aplicación contable que permita ejecutar diversos cálculos de los resultados económicos generados por los esfuerzos empresariales realizados para lograr una restauración o preservación con el fin de alcanzar un equilibrio ambiental, para ello es necesario que las organizaciones reconozcan los costos y gastos incurridos en la conservación del patrimonio natural (Robles, 2017). 
En este contexto, se define al costo ambiental como los materiales y recursos necesarios para la producción en diversas industrias encaminadas a satisfacer las necesidades de la sociedad en general, los mismos costos que son provenientes de la naturaleza y que deben ser medidos, controlados, clasificados y evidenciados en los estados financieros con el fin de optimizarlos y buscar nuevas acciones o formas de producción amigables con el medio ambiente, ligadas de manera directa con la responsabilidad social de los clientes o consumidores, quienes dan referencias positivas de los productos o servicios que adquieren y el impacto que generan las empresas en el medioambiente al ser responsables (Carreño, Erazo, Narváez, \& Moreno, 2020).

Es así como, las industrias deben hacer frente a través de la adopción de estructuras amigables, en cuanto a la optimización de energía, agua, materiales y demás, puesto que los escasos recursos son utilizados en diversos procesos productivos con el fin de crear bienes y distribuir a la sociedad, lo que a su vez conlleva a una cultura cada vez más consumista y exigente; así también, las empresas tienen la responsabilidad de medir y registrar las acciones emprendidas a favor del medio ambiente y brindar información para la toma de decisiones internas y externas, así también de forma paralela debe existir una organización de nivel superior que controle el cumplimiento de normas con el fin de mitigar las actividades dañinas con el medio ambiente.

Sobre la base de las ideas expuestas, investigando la adopción de la gestión de costos ambientales en el Ecuador, se evidencia que gran parte de las organizaciones no tienen definidos sus costos ambientales y no los tienen desarticulados por actividades, procesos o productos, lo que impide su adecuada y eficiente gestión, es por eso, que se requiere plantear estrategias para mejorar la información específica de los costos de operación y sea fuente de investigación y desarrollo a futuro. El éxito de las estrategias implementadas depende del trabajo realizado en conjunto por todos los integrantes de la organización y de los acuerdos con los administradores corporativos respecto a la información recabada a cerca del medio ambiente, es importante tener una visión compartida. 
Luego de analizar los procesos de la contabilidad ambiental y avanzar en la explicación teórica de costos y gastos ambientales, se trae a contexto la auditoría ambiental pues es importante profundizar este punto de tal manera que ambas se conviertan en mecanismos de mitigación de los impactos ambientales generados por las diversas actividades productivas y que éstas se puedan reorientar bajo principios de sostenibilidad.

Es necesario resaltar que la función de la auditoría se materializa no solo en la verificación de prácticas, sino en el hecho de institucionalizar esfuerzos por reconocer las intenciones organizacionales que transforman el modus operandi de las unidades productivas neutralizando la relación empresa, ser humano y entorno ambiental, en consecuencia, la auditoría ambiental brinda información acerca de los productos realizados bajo normas productivas organizadas y sustentables a la sociedad que los consume y da señales a cerca del cumplimiento de estándares de calidad (Ospina \& Arango, 2017). Por tal motivo, la eficiencia con la que disminuyen las brechas asimétricas de la información entre productores y clientes determinará su eficiencia; es así como se genera un cambio en la conciencia colectiva con el fin de plantear una producción sostenible y obtener una buena calidad de vida y la conservación del medio ambiente.

En conclusión, el mercado es cada vez más exigente y la globalización debe ir acompañada de buenas prácticas empresariales las cuales deben ser concientizadas en las empresas y desarrollar con el paso del tiempo una adecuada responsabilidad social y ambiental mediante nuevas estrategias consolidadas y diversas herramientas, es decir la contabilidad requiere asentar en la organización el concepto de desarrollo sostenible a través de la inclusión de información transparente respecto a su comportamiento ambiental (Galvis \& Guevara, 2019). 


\section{Mejores prácticas contables adoptadas en las empresas de Latinoamérica}

La contabilidad ambiental con la finalidad de presentar resultados financieros que respalden la toma de decisiones planteadas en sus inicios por la contabilidad financiera, debe contener partidas que expliquen los resultados económicos de las operaciones derivadas respecto a la conservación y mejoramiento del ecosistema para conseguir sostenibilidad a través de los años; lo que sin duda significa un gran desafío para la contabilidad, la cual se debe basar en la observación, clasificación, valoración y exposición de las partidas contables que tienen incidencia con el medio ambiente (Colmenares, Valderrama, \& Adriani, 2015).

En este contexto, se ha tratado de evidenciar la importancia de adoptar políticas contables ambientales en las empresas de Latinoamérica con el fin de alcanzar la sostenibilidad, ya que la naturaleza y el ambiente tienen su realidad compleja y no se puede enfocar solo a la obtención de ganancias monetarias. Según el autor (Ccopa, 2014), el capitalismo y sus teorías acumulativas y de crecimiento descontrolado hacen que entren en contradicción las condiciones normales de producción y se sigan las vías más rápidas que lastimosamente no son las más amigables con el ecosistema.

Ante lo expuesto, es preciso mencionar que el Ecuador es un país con recursos hídricos abundantes, en donde la interacción del hombre con la biosfera ocasiona dificultades relacionadas con la contaminación del agua, el suelo y el aire, elementos que inciden en la humanidad y en la pérdida de la flora y fauna autóctona los mismos que son indispensables para la ecología y la bioética en el territorio ecuatoriano, al respecto, en América Latina el reconocimiento del agua como derecho humano fundamental y como sujeto de derechos a la naturaleza en el marco constitucional del pluralismo jurídico forma parte de precautelar la seguridad hídrica que garantice su efectiva distribución y ejercicio de los derechos (Sánchez \& Tello, 2019), más aún cuando los ciclos normales y naturales del agua han sido considerados como un bien desde los pueblos originarios en el sumak kwasay, por lo que es necesario su protección adecuada, a fin de evitar procesos 
contaminantes que incidan en la cantidad, calidad y distribución de este elemento vital (Sánchez \& Tello, 2019).

Para mitigar lo mencionado en el párrafo anterior, así como enlazar la contabilidad pura con el tratamiento ambiental para generar criterios de cambios y reestructurar políticas basadas en normas internacionales, (Galvis \& Guevara, 2019) realizan la propuesta de los siguientes cambios que se deben presentar en el ámbito contable para mitigar los daños:

- Es necesario que la contabilidad salga de los tradicionales marcos teóricos y se de paso a una interdisciplinariedad con las ciencias naturales, sociales y biofísicas, esto con el fin de alcanzar el desarrollo de nuevos modelos eficientes y nuevas herramientas aplicables en la preocupante situación actual, para así crear relaciones sólidas que se manejen con un enfoque simplificado.

- Reformular la valoración y medición respecto a las variables ambientales; es decir, se debe determinar un adecuado criterio de medición para los recursos naturales, bienes ambientales y los impactos sociales de la producción; se debe medir y controlar cada cierto periodo de tiempo el impacto de la empresa en el medio ambiente, así se puede definir evaluaciones anuales, semestrales, mensuales, entre otras.

- Poner un freno al interés único de crecimiento financiero, hace referencia al capitalismo que busca la acumulación de bienes y riquezas sin tener consciencia que los procesos productivos están dejando secuelas irreversibles en el entorno y que a largo plazo cobrará un valor más alto todavía, por lo cual existe el enfoque en la sostenibilidad ambiental, con la finalidad de crear e innovar procesos eco amigables y más eficientes. 
Otro aporte esencial no sólo es el adaptar la contabilidad ambiental, sino que debe venir desde la educación en las instituciones, por lo que, el ámbito académico ecuatoriano tiene un gran desafío por delante, por cuanto se requiere incluir en la metodología nuevas investigaciones, teorías y herramientas que consoliden el aprendizaje y a futuro puedan ser aplicadas en la organización, así como en Colombia que ya ha tenido sus inicios más o menos a partir de la década de los 90 en estudios relacionados con esta temática contable (Galvis \& Guevara, 2019). Es imprescindible tratar la forma de cómo la contabilidad ambiental en teoría es impartida en la educación, y el peso e importancia que tiene, puesto que se exige se tome mayor consciencia y no sólo sea un estudio teórico sino se lleve a la práctica desde las universidades, posgrados, entre otros y se extienda a las empresas de América Latina.

Por otro lado, tomando como ejemplo a México, se analiza que cuenta con dos regímenes de mediciones y cálculos para alcanzar la sostenibilidad: por una parte el sistema de cuentas económicas y ecológicas de México, que incorpora mediciones ambientales en las cuentas nacionales, y por otra parte, cuenta con indicadores de desarrollo sustentable que dan cuenta de variables económicas, financieras, ecológicas, sociales e institucionales (Rivera \& Foladori, 2006).

De igual manera se analiza el rol del contador y su postura para ejecutar su función, y se trae a contexto Venezuela, en el análisis se determina que a pesar de que existen normas regulatorias en el Estado venezolano referente a la materia ambiental en la contabilidad, existe desconocimiento en los profesionales más aún al momento de aplicar en las organizaciones, por cuanto, para evitar una serie de inconvenientes se sigue manejando la contabilidad en su forma tradicional, es entonces cuando se evidencia un llamado divorcio entre el contador y los tratamientos financieros respecto al impacto ambiental (Álvarez, Urbina, Guerrero, \& Castro, 2009); este análisis determina la necesidad de encadenar estas teorías desde la formación de los profesionales para que se vean reflejados en la práctica de su oficio, así como también se debe brindar capacitaciones 
de la materia a nivel mundial para alcanzar la unificación de medidas y aplicación a nivel global a favor de la sostenibilidad medioambiental.

\section{MÉTODO}

La presente investigación fue de tipo descriptiva no experimental por cuanto no existió manipulación alguna de las variables, así también se utilizó un diseño transversal, por cuanto se realizó el levantamiento de la información por única vez; se consideró los principios de la contabilidad ambiental a través de la historia, así como su amparo en las normas y reglamentaciones que las rigen en este caso las NIIF de forma específica la norma 6 y al mismo tiempo explicó por qué ocurre este fenómeno y en qué condiciones se dio.

Se empleó el método analítico-sintético, pues permitió analizar las normas sobre las cuales reposa la contabilidad ambiental desde sus inicios hasta la actualidad y como se ve relacionada de forma directa con el cuidado o destrucción del medio ambiente. La técnica de recolección de datos fue la encuesta, para ello se elaboró un cuestionario con 20 ítems orientados a valorar las buenas prácticas contables-ambientales y el registro, evaluación y comunicación de la actuación medioambiental de las empresas, este instrumento se sometió a un proceso de evaluación por vía de expertos, sus resultados fueron analizados a través del coeficiente de alpha de crombach (tabla 1), que es considerado como un modelo de consistencia interna, sustentado en el promedio de correlación entre los ítems, obteniéndose una fiabilidad de 0.81 que significa, que los ítems se están midiendo de manera consistente. En lo posterior, se aplicó la encuesta al universo de estudio conformado por 3 grandes organizaciones: Induglob, Ecoline y Ecogas. 


\section{RESULTADOS}

A continuación se presenta el resultado de las encuestas aplicadas a las diferentes empresas de línea blanca de la provincia del Azuay-Ecuador que fueron realizadas con la finalidad de obtener información acerca del conocimiento y aplicación de la contabilidad ambiental así como su relación con el medio ambiente dependiendo de las actividades económicas ejecutadas y la gestión contable que desarrolla cada una:

Conocimiento de la contabilidad ambiental: partiendo del conocimiento de la contabilidad ambiental y lo que implica, se obtuvo que el $67 \%$ de los empleados de las empresas encuestadas no tienen conocimiento acerca de un concepto claro de la contabilidad ambiental o su manera de aplicación, ejecución y presentación a través de los estados financieros, lo que se deriva en que no se aplica contabilidad ambiental en las empresas productoras de línea blanca.

Objetivo de la contabilidad ambiental: para una aplicación de la contabilidad ambiental fue importante el evaluar si se tiene el conocimiento de cuál es el objetivo de la contabilidad ambiental y se obtuvo que el $67 \%$ de los empleados de las empresas encuestadas relacionan de manera directa la contabilidad ambiental con promover el buen uso de los recursos naturales y una de las tres empresas consideran que ninguno de los ítems mencionados se relaciona con la contabilidad ambiental, entre los cuales estaba como opción la reforestación, cambios de procesos productivos y evaluaciones ambientales.

Preocupación de las empresas por el medioambiente: se analizó la percepción por parte de los empleados, en cuanto a que, si las empresas en las que laboran muestran interés o preocupación por la parte ambiental o el daño reversible o irreversible que percibe el medio ambiente debido a las actividades de sus empresas, al respecto, se obtuvo que el $67 \%$ de los empleados consideran que sí existe preocupación por las actividades y su influencia directa, mientras que, el $33 \%$ restante percibe que no existe preocupación alguna de las empresas por el ecosistema y medio ambiente. 
Adopción de la contabilidad ambiental: referente a adoptar nuevas medidas o nuevas prácticas contables amigables con el medio ambiente, resultó que un $33 \%$ considera que la empresa donde labora está lista para adoptar la contabilidad ambiental, es decir, tienen la iniciativa y predisposición de implementar la contabilidad ambiental, mientras que, el $67 \%$ restante consideran que no estarían listos para adaptar nuevos cambios a menos que se reciba una formación completa ya sea interna o externa para adaptar la contabilidad ambiental.

Apoyo de entidades externas: referente al apoyo externo, el $67 \%$ de los empleados de las empresas encuestadas mencionan que no cuentan con apoyo de entidades externas para un mejor desarrollo ni con auditorías que garanticen las buenas prácticas medio ambientales, en este sentido, se percibe una ligera despreocupación o presión de los entes ajenos a la empresa para ejecutar una sostenibilidad ambiental que vaya a la par con las actividades empresariales pues mencionan que desde las máximas organizaciones de la nación no hay material reglamentario claro para su aplicación.

Incentivos a colaboradores para ser parte de actividades ecológicas: el $67 \%$ de los empleados de las empresas encuestadas mencionan que algunas veces si existe motivación a los colaboradores para ser parte de actividades ecológicas no permanentes, es decir, se ejecutan campañas en periodos específicos por ejemplo se realizaron actividades y concursos por el día del reciclaje y premiaron la adecuada separación de desechos.

Campañas para el uso de medios de transporte alternativos: es interesante el resultado de la presente pregunta referente a que si existe o no campañas para promover el uso alternativo de medios de transporte para dirigirse a sus labores diarias y actividades cotidianas, por cuanto el $100 \%$ de los empleados de las empresas encuestadas mencionó que no existe dicha motivación ni campañas internas para este cambio, sin embargo, mencionan estar dispuestos a ser parte de este cambio o inclusive 
compartir el medio de transporte entre compañeros que tengan sus viviendas en zonas aledañas.

Tratamiento contable de un estudio de impacto ambiental: mediante esta pregunta se analizó como se reconocería contablemente un estudio de impacto ambiental, al respecto, una de las empresas respondió que se reconocerá como un activo intangible y las otras empresas mencionaron que se registraría como un gasto, este resultado deja en evidencia la falta de consenso y conocimiento de las cuentas ambientales y su tratamiento contable.

Opciones de mejora en procesos: una de las empresas considera que sí se pueden realizar mejoras en los diferentes procesos productivos para ser amigable con el medio ambiente y dos de las empresas consideran que no hay oportunidades de mejora en sus procesos internos o al menos en ese momento no tenían claro que se podría mejorar.

Tratamiento de aceites provenientes de las maquinarias: El área contable de ninguna de las empresas tienen conocimiento del tratamiento de los aceites provenientes de las maquinarias y la afectación al medio ambiente que provoca su mal uso. Esto deja en evidencia que las empresas de línea blanca localizadas en la provincia del Azuay no han emprendido acciones para mitigar los daños medioambientales ocasionados por sus procesos de producción. 


\section{PROPUESTA}

A partir de los resultados obtenidos, se diseña una guía para la adopción de la contabilidad ambiental en las empresas de línea blanca de la provincia del Azuay, sustentada en cuatro elementos que se exponen en la figura 1.

\section{Contabilidad Ambiental \\ EMPRESAS L I N EA \\ $B$ L A N C A}

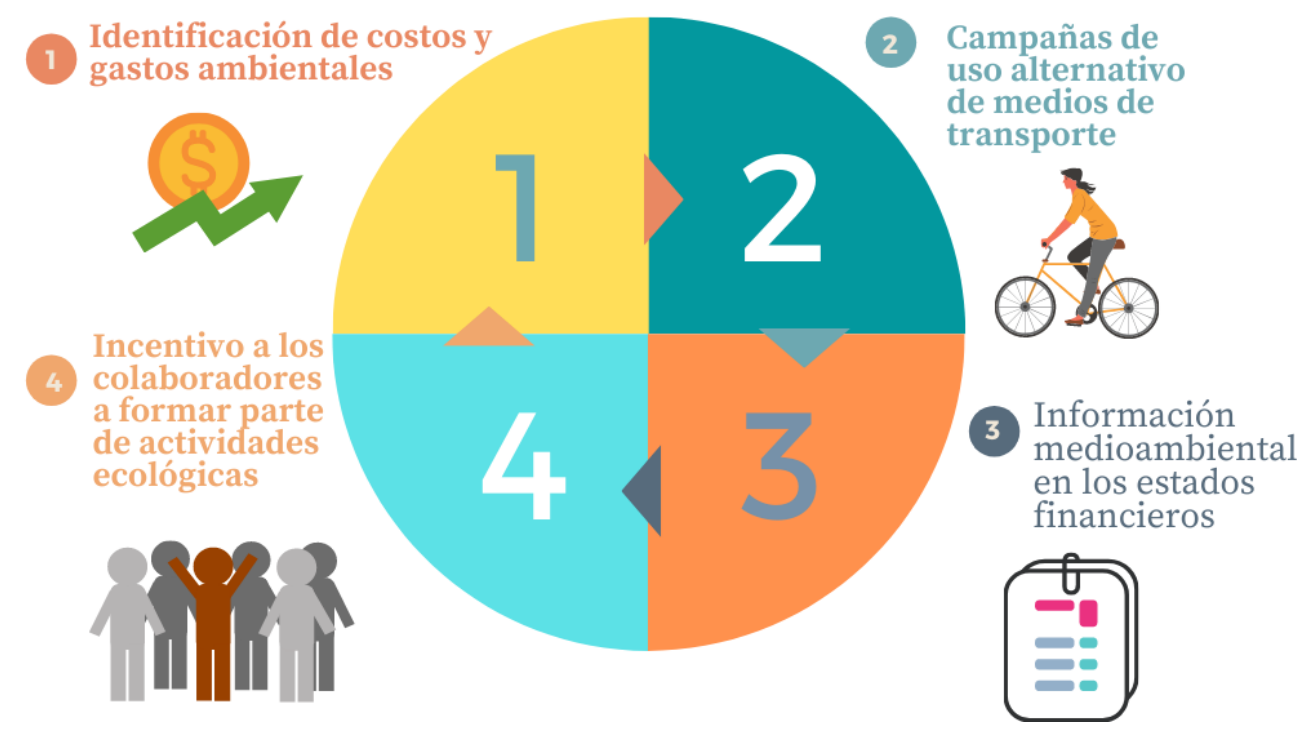

Figura 1. Guía para la implementación de contabilidad ambiental en las empresas de producción de línea blanca en la provincia del Azuay.

\section{Identificación de costos y gastos ambientales}

\section{Gastos ambientales}

Determinar gastos ambientales es definir e identificar los mismos que se incurren en busca de la protección ambiental, es decir, proteger el medio ambiente o tratar de restaurar el mismo con diferentes actividades como buscar otros sistemas alternativos de energía eléctrica, un adecuado reciclaje de los desechos o reutilización de los mismos 
mediante procesos productivos adicionales, ayudar en la reforestación, uso consciente del agua, entre otros.

\section{Costos ambientales}

Se refieren a costos internos o externos los mismos que poseen relación directa con los efectos dañinos causados al medio ambiente, es decir su finalidad es la inversión en protección ambiental que plantea la creación de prevención y control para resarcir daños originados en las compañías y que afectan de manera directa a la sociedad, la propuesta va ligada a buscar métodos de producción más ecoeficientes y amigables, es decir seguir produciendo con un precio competitivo que satisfaga las necesidades de los clientes con un impacto mínimo o nulo ambiental.

Los costos ambientales se deben clasificar de la siguiente manera:

- Costos de prevención ambiental: son las actividades que se realizan con el fin de prevenir la producción de desechos o residuos contaminantes que ocasionen daños al medio ambiente y para alcanzar este fin se puede realizar una adecuada evaluación y selección de proveedores, auditorías de riesgos ambientales, obtención de certificaciones ISO, adecuado reciclaje de productos, entre otros.

- Costos de detección ambiental: su objetivo es determinar si se cumple con los estándares ambientales adecuados que abarcan procesos, actividades y productos, para los antes mencionados rigen las leyes de los gobiernos y políticas ambientales desarrolladas por la administración y las herramientas a utilizar serían las pruebas y mediciones de los niveles de contaminación, inspección o auditorías de las actividades ambientales, entre otros.

- Costos de fallas ambientales internas: son los costos que se desencadenan de malos procesos productivos y generan contaminantes y residuos que se liberan al medio ambiente sin ninguna restricción o tratamiento preliminar como es el caso de los aceites de las maquinarias, desechos tóxicos, equipos que producen fuerte 
Universidad Nacional Experimental Francisco de Miranda (UNEFM). Santa Ana de Coro. Venezuela

Alexandra Estefanía Sarmiento-Figueroa; Diego Vinicio Orellana-Bueno; Pablo Javier Perez-Jara contaminación, entre otros. De otra forma, pretende manejar de la manera adecuada todo desecho producido y canalizar hasta su correspondiente tratamiento.

- Costos de fallas ambientales externas: se refiere a la descarga o liberación de contaminantes y residuos hacia el ambiente y se plantea que se debe contar con un plan de tratamiento para los mismos, o a su vez ser reemplazados con productos menos agresivos para el medio ambiente así como también mejorar los procesos productivos y que los mismos no emanen gases dañinos para el ambiente y no repercuta en la salud de los ciudadanos.

\section{Campañas de uso alternativo de medios de transporte}

Desarrollar campañas internas y externas de ser posible para fomentar y promover el uso de transporte compartido o sistemas de medios ecológicos, lo cual es una forma responsable y sostenible de brindar protección al medio ambiente.

Tiene como finalidad reducir o minimizar el uso de vehículos y otros medios de transporte que generan gases contaminantes al medio ambiente, así como también se busca fomentar y promover el transporte público, transporte compartido o el uso de otros medios de transporte no contaminantes como es el uso de la bicicleta y de ser posible la caminata si el lugar de trabajo queda a una corta distancia, ya que de forma paralela ayuda a la salud; se deja claro que el compartir vehículo incide en una reducción significativa de gases contaminantes, contaminación acústica, ahorro en gastos de traslado, menos tráfico, más estacionamientos, entre otros. 
A continuación un ejemplo de campaña que se podría utilizar en las empresas.

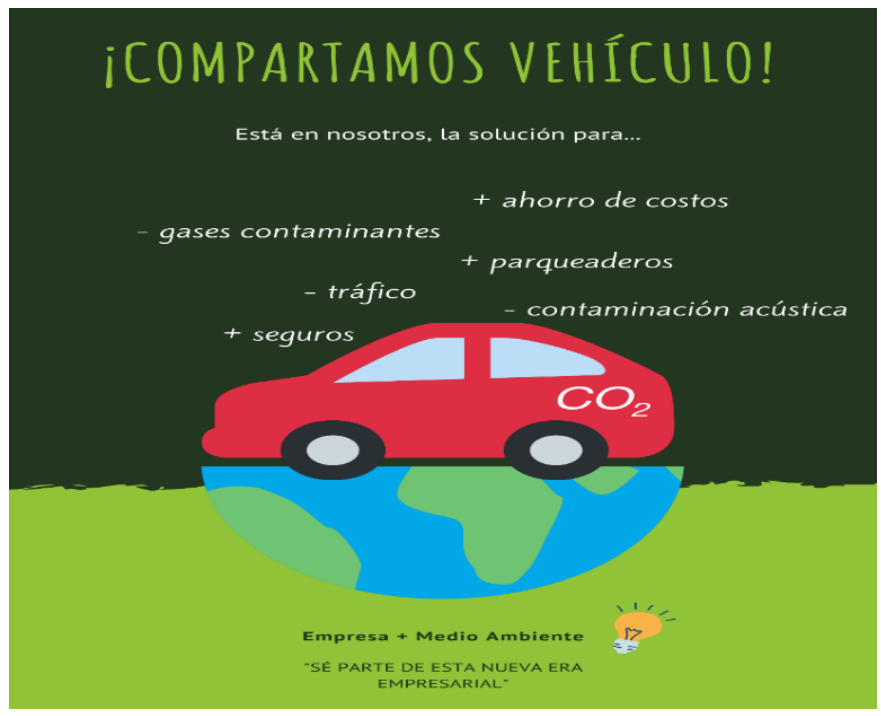

Figura 2. Campaña para impulsar el uso de vehículo compartido.

\section{Información medioambiental en los estados financieros.}

Esta propuesta busca presentar información medioambiental en los estados financieros y se propone añadir la información a continuación:

a) Inversiones que se realicen ya sea en bienes de activo fijo o llamados activos ambientales, los mismos se deberán presentar separado de los otros activos, adicional se identificarán los activos ambientales con los responsables de la gestión ambiental a manera de ejemplo se mencionan los siguientes a continuación:

- Partidas: inventarios, inversiones, propiedad planta y equipo.

- Subpartidas: capital natural o recursos renovables, espacios físicos comprometidos para el desarrollo de la política ambiental, procesos tecnológicos favorables al entorno, maquinarias y equipos que reduzcan los efectos contaminantes. 
b) Obligaciones contraídas para la protección del medio ambiente o también llamados pasivos ambientales, se cita las deudas en materia ambiental o compromiso asumido con el objetivo de evitar, reducir o reparar el impacto medioambiental identificando los siguientes componentes:

- Pasivo cierto: se identifican de forma clara la cuantía de la obligación contraída.

- Pasivo contingente: no se determina con certeza, es decir, está condicionado a la ocurrencia o no de sucesos que se den a futuro y no se predice hasta que sucedan.

c) Provisiones generadas cuando haya obligación por daños al ambiente las mismas que en efecto poseen incertidumbre acerca de su cuantía o vencimiento y que serán utilizadas para cubrir los desembolsos para los cuales fueron en sus inicios reconocidas, se podrían presentar por los siguientes motivos:

- Administración de desechos y reciclado.

- Restauración del ecosistema.

- Limpieza de sitios contaminados.

- Medidas de protección ambiental.

- Riesgos y gastos ambientales.

- Cese de actividades (restauración del daño ambiental ocasionado una vez finalizadas las actividades productivas).

d) Costos en que incurra la empresa, bien de forma obligatoria o voluntaria, y que tengan un carácter medioambiental. (detalle en elemento 1)

e) Riesgos y contingencias en que la empresa se encuentre envuelta, derivados de la incidencia de su actividad en el entorno.

f) Cualquier otra información derivada de la interacción empresa o medio ambiente, bien sea cuantitativa, cualitativa o financiera 
Revista Interdisciplinaria de Humanidades, Educación, Ciencia y Tecnología

Año VI. Vol. VI. N². Edición Especial II. 2020

Hecho el depósito de ley: pp201602FA4721

ISSN-L: 2542-3029; ISSN: 2610-802X

Universidad Nacional Experimental Francisco de Miranda (UNEFM). Santa Ana de Coro. Venezuela

Alexandra Estefanía Sarmiento-Figueroa; Diego Vinicio Orellana-Bueno; Pablo Javier Perez-Jara

En esta propuesta se añade el uso de indicadores de evaluación de la sostenibilidad ambiental, en la tabla 2 se presentan algunos ejemplos:

Tabla 1.

Indicadores contables para la gestión ambiental.

\begin{tabular}{|c|c|c|}
\hline \multicolumn{3}{|c|}{ Indicadores del Activo } \\
\hline Indicadores & Fórmula & Interpretación \\
\hline $\begin{array}{l}\text { Rentabilidad sobre activos } \\
\text { ambientales }\end{array}$ & $\begin{array}{l}=\text { Utilidad bruta/activo total } \\
\text { ambiental }\end{array}$ & $\begin{array}{l}\text { Mide la rentabilidad de los activos ambientales } \\
\text { de una empresa, estableciendo para ellos una } \\
\text { relación entre los beneficios netos y los activos } \\
\text { totales ambientales. }\end{array}$ \\
\hline \multicolumn{3}{|c|}{ Indicadores del Pasivo } \\
\hline Indicadores & Fórmula & Interpretación \\
\hline Endeudamiento & $\begin{array}{l}=\text { Pasivo ambiental/activo } \\
\text { total }\end{array}$ & $\begin{array}{l}\text { Por cada dólar invertido en activos ambientales, } \\
\text { cuánto está financiado por terceros. }\end{array}$ \\
\hline \multicolumn{3}{|c|}{ Indicadores de Costos } \\
\hline Indicadores & Fórmula & Interpretación \\
\hline Contribución marginal & $\begin{array}{l}\text { =Ingresos operacionales - } \\
\text { costos y gastos variables } \\
\text { ambientales }\end{array}$ & $\begin{array}{l}\text { Se considera a la contribución marginal como la } \\
\text { fracción de ventas que contribuye a cubrir los } \\
\text { costos fijos, es la cantidad que cada venta } \\
\text { unitaria le agrega a la ganancia se le conoce } \\
\text { como la pendiente de la línea de ganancia. }\end{array}$ \\
\hline \multicolumn{3}{|c|}{ Indicadores de Gastos } \\
\hline Indicadores & Fórmula & Interpretación \\
\hline $\begin{array}{l}\text { Gasto total en protección ambiental } \\
\text { respecto a la utilidad bruta }\end{array}$ & $\begin{array}{l}\text { =Gastos en protección } \\
\text { ambiental/utilidad bruta }\end{array}$ & $\begin{array}{l}\text { Mide la porción de gastos invertidos en la } \\
\text { protección ambiental respecto a la utilidad } \\
\text { bruta de la empresa. }\end{array}$ \\
\hline $\begin{array}{l}\text { Gasto en gestión de residuos } \\
\text { respecto al gasto total }\end{array}$ & $\begin{array}{l}=\text { Gastos en gestión de } \\
\text { residuos/gasto total }\end{array}$ & $\begin{array}{l}\text { Mide la porción de gasto ambiental respecto al } \\
\text { gasto total de la empresa. }\end{array}$ \\
\hline $\begin{array}{l}\text { Gasto en investigación y desarrollo } \\
\text { ambiental respecto al gasto total }\end{array}$ & $\begin{array}{l}=\text { Gastos en investigación y } \\
\text { desarrollo ambiental/gasto } \\
\text { total }\end{array}$ & $\begin{array}{l}\text { Mide la porción de gasto generado por } \\
\text { investigación y desarrollo respecto al gasto total } \\
\text { de la empresa. }\end{array}$ \\
\hline
\end{tabular}




\section{Incentivos a los colaboradores a formar parte de actividades ecológicas.}

Esta propuesta busca comprometer a los colaboradores con el cuidado medioambiental tanto de las empresas como en su entorno y ello se logrará mediante lo siguiente:

- Creación de un ambiente favorable: busca estimular el desarrollo de las actividades y recompensar a los empleados que ejecutan las buenas prácticas con el medio ambiente, es importante tener un canal abierto de comunicación para propuestas y sugerencias.

- Capacitaciones: imprescindibles para crear una cultura nueva sostenible y amigable con el medio ambiente, es por ello que, los empleados deben entender su papel e importancia en la empresa y conseguir el compromiso para llevar a cabo las diferentes actividades; este paso se puede ejecutar mediante reuniones enriquecedoras, conferencias, videos, entrenamientos, paseos, entre otros, que no requieren de un flujo de dinero.

- Campañas de concienciación: buscan llegar a cada uno de los colaboradores para enfatizar la importancia de las pequeñas acciones realizadas por cada uno de ellos en su día a día y reflexionar acerca del impacto positivo que tendrían las mismas en el medio ambiente y es tan sencillo como separar la basura de su puesto de trabajo y colocar en el basurero correcto, o también reducir el gasto de consumo de luz o teléfono, uso adecuado de los interruptores, entre otros.

\section{CONCLUSIÓN}

En el estudio de la contabilidad ambiental a lo largo del tiempo se han observado aportes y avances significativos por cuanto se busca mitigar el daño ocasionado en el medio ambiente por las diversas actividades humanas, enfocado en esta ocasión en las empresas de producción de línea blanca de la provincia del Azuay-Ecuador con el fin de alcanzar la sostenibilidad económica y ambiental. 
Para llevar a cabo un proceso exitoso se requiere de la auditoría ambiental, que certifique el cumplimiento de estándares y ponga su mayor esfuerzo en la búsqueda de mejorar la forma en que operan las organizaciones, es decir, sus procesos productivos que deben basarse en una normativa que regule las buenas prácticas contables.

Respecto a los resultados obtenidos en este estudio se evidenció que las empresas de línea blanca del Azuay-Ecuador no aplican contabilidad ambiental en las diferentes cuentas de los estados financieros ni se rigen a una normativa internacional y no tienen un control o auditoría interna que norme las buenas prácticas ambientales.

Al mismo tiempo, las empresas presentan la predisposición de aplicar la contabilidad ambiental así como también son conscientes que no están preparados para hacerlo, por lo que requieren del apoyo de entidades externas que den pautas claras y sobretodo capacitación de cómo se debería aplicar, así también se menciona que los empleados no tenían conocimiento de la finalidad de los aceites provenientes de las maquinarias y su tratamiento.

Por otra parte, para comenzar a adoptar la contabilidad ambiental en cualquier empresa se requiere de un estudio preliminar para conocer la situación actual de la misma y definir el punto al que se quiere llegar, para lo cual se requiere de cambios y acciones que se deben establecer de forma específica con periodos de tiempos, actividades, responsables, cronograma, entre otros, con el fin de revolucionar sus procesos productivos y actividades cotidianas siendo sostenibles de forma económica y ahora de también con el ambiente.

La guía para implementar la contabilidad ambiental propuesta para las empresas de producción de línea blanca, se sustenta en la identificación de costos y gastos ambientales, campañas de uso alternativo de medios de transporte, información medioambiental en los estados financieros e incentivos a los colaboradores a formar parte de actividades ecológicas, en este sentido, la guía constituye una herramienta esencial para el adecuado registro, evaluación y comunicación de la actuación 
medioambiental de estas empresas, cabe resaltar que el tratamiento contable se deberá efectuar bajo estándares internacionales considerando los parámetros de las NIIF y de la Ley de Régimen Tributario Interno (LORTI) vigente en Ecuador.

\section{REFERENCIAS CONSULTADAS}

Álvarez, Romer, \& Urbina, Luisa, \& Guerrero, Francisco, \& Castro, Josefa (2009). Contabilidad de gestión ambiental en el ejercicio de la profesión del contador público en el estado Zulia. Revista de Ciencias Sociales (Ve), XV(3),499-508.

Arguello, M. B. (2018). La problemática de la contabilidad ambiental. [The problem of environmental accounting]. Revista Publicando, 5 No 15. (2).

Bischhoffshause, W. v. (2016). Una visión general de la contabilidad ambiental. [An overview of environmental accounting]. Contaduría universidad de Antioquía. doi:https://url2.cl/IMHCF

Caraballo, P., \& Barros, N. (2011). Ecología y bienestar humano. [Ecology and human well-being]. Revista colombiana de ciencia animal, 141-147. doi:https://url2.cl/PQXaP

Carreño, M. J., Erazo, J. C., Narváez, C. I., \& Moreno, V. P. (2020). La responsabilidad social en las empresas camaroneras. Revista Arbitrada Interdisciplinaria Koinonía, 5(10), 455-482. http://dx.doi.org/10.35381/r.k.v5i10.702

Ccopa, C. A. (2014). Evaluación crítica de la contabilidad ambiental empresarial en el Perú. [Critical evaluation of corporate environmental accounting in Peru]. Comuni@cción. doi:https://url2.cl/Xys1M

Colmenares, L., Valderrama, Y., \& Adriani, R. (2015). Representación contable desde la perspectiva del impacto ambiental empresarial en el contexto del desarrollo de actividades industriales en Latinoamérica. [Accounting representation from the perspective of business environmental impact in the context of the development of industrial activities in Latin America]. Cuadernos de contabilidad. doi:https://url2.cl/zSzZL

Díaz, S. G. (29 de Noviembre de 2019). Componentes para analizar los resultados de una contabilidad ambiental. [Components to analyze the results of an environmental accounting]. Proyecciones, 29. doi:https://url2.cl/GwXe4 
Galvis, M. R., \& Guevara, A. S. (2019). Una mirada a la contabilidad ambiental en Colombia desde las perspectivas del desarrollo sostenible. [A look at environmental accounting in Colombia from the perspectives of sustainable development]. Facultad de ciencias económicas, 87-106. doi:https://url2.cl/Aak6W

Hernández, Y. M. (2016). Contabilidad de costos ambientales en la industria cámica, recomendaciones para su desarrollo. [Accounting of environmental costs in the camic industry, recommendations for its development]. Ciencias Holguín, 1-14. doi:https://url2.cl/wi7Md

Jara, A. A., Díaz, M. P., \& Morales, E. L. (2017). La contabilidad ambiental en empresas del cantón Morona, provincia de Morona Santiago, Ecuador. [Environmental Accounting in companies in the Morona Canton, Morona Santiago Province, Ecuador]. Revista publicando, 213-237. doi:https://url2.cl/VWLXD

Jiliberto, R. R., Urbani, P., Narváez, L. G., Barry, P. R., Montoya, F. E., \& Medina, M. (2013). Identificación de especies ecológicamente relevantes para la evaluación de riesgo ecológico: una propuesta desde la ecología teórica. [Identification of ecologically relevant species for ecological risk assessment: a proposal from theoretical ecology]. Revista chilena de historia natural, 86(1), 2131. https://dx.doi.org/10.4067/S0716-078X2013000100003

López, C. A., Rodríguez, L. M., \& Gómez, D. F. (2019). Integración de la dimensión ambiental al sistema de información financiera de la fábrica de piensos cienfuegos. [Integration of the environmental dimension into the financial information system of the cienfuegos feed mill]. Observatorio de la economía latinoamericana., 20. doi:https://url2.cl/s4Qfz

Luna, M. C. (2016). Relación de la contabilidad ambiental y las normas internacionales de información financiera: mini revisión de literatura. [Relationship of environmental accounting and international financial reporting standards: mini literature review]. Pontificia universidad javeriana, 55. doi:https://url2.cl/Bbcb8

Macías, G. P., \& Roig, M. C. (2018). La contabilidad ambiental como herramienta de gestión para el turismo sostenible.[Environmental accounting as a management tool for sustainable tourism]. Cofin Habana, 12(1), 124-146. 
Ramírez Ospina, Duván Emilio, \& Andrade Arango, Yamilhet. (2017). Aporte de la contabilidad y la auditoría desde una perspectiva ambiental. Textual: análisis del medio rural latinoamericano, (69), 2741. https://doi.org/10.5154/r.textual.2017.69.002

Porras, D. B. (2018). La contabilidad ambiental en el desarrollo sustentable del sector turístico de la provincia de Tungurahua. [Environmental accounting in the sustainable development of the tourism sector in the province of Tungurahua]. doi:https://url2.cl/PTYsH

Rivera, P., \& Foladori, G. (2006). Reflexiones sobre la contabilidad ambiental en México. [Reflections on environmental accounting in México]. Economía sociedad y territorio. doi:https://url2.cl/gWm1U

Rizo, M. A., \& Collado, N. V. (2017). La contabilidad y la actividad medio ambiental de la industria ronera en Cuba: caso de estudio empresa mixta Havana Club International S.A-Ronera San José. [Accounting and environmental activity of the rum industry in Cuba: case study of a joint venture Havana Club International S.ARonera San José]. Contabilidad y negocios.

Robles, A. L. (2017). Importancia y aplicabilidad de la Contabilidad Ambiental en las empresas colombianas. [Importance and applicability of Environmental Accounting in Colombian companies]. Universidad militar Nueva Granada, 39.

Rodríguez, D. H. (2011). Contabilidad ambiental: Fundamentos epistemológicos, humanistas y legales. [Environmental accounting: Epistemological, humanistic and legal foundations]. Económicas cuc, 35-44.

Sánchez, A. A., \& Tello, L. L. (2019). La contaminación ambiental en los acuíferos de Ecuador. [Environmental pollution in the aquifers of Ecuador]. Visión contable.

Sánchez, F. J., \& Pedrajas, A. P. (2010). La comprensión de conceptos de ecología y sus implicaciones para la educación ambiental. [Understanding ecology concepts and their implications for environmental education]. Revista eureka sobre enseñanza y divulgación de las ciencias, 271-285. 